\title{
SURFACES WITH A PARALLEL ISOPERIMETRIC SECTION
}

\author{
BY BANG-YEN CHEN \\ Communicated by Philip Hartman, November 20, 1972
}

This announcement is a continuation of Chen [1] (also, Yau [3]). We shall present additional theorems relating surfaces in a space form with a parallel normal section.

Let $M$ be a surface in an $m$-dimensional Riemannian manifold $R^{m}$ with the induced normal connection $D$. For a unit normal section $\xi$ on $M$ (that is, a unit normal vector field of $M$ in $R^{m}$ ), let $A_{\xi}$ be the second fundamental tensor with respect to $\xi$; if we have $D \xi=0$ identically, then $\xi$ is called a parallel section; if the trace of $A_{\xi}$ is constant (respectively, zero), then $\xi$ is called an isoperimetric section (respectively, minimal section) on $M$; if the determinant of $A_{\xi}$ is nowhere zero, then $\xi$ is called a nondegenerate section; if $A_{\xi}$ vanishes identically, then $\xi$ is called a geodesic section; and if $A_{\xi}$ is not proportional to the identity transformation everywhere, then $\xi$ is called a umbilical-free section.

THEOREM 1. Let $M$ be a closed surface in an m-dimensional Riemannian manifold $R^{m}$ of constant sectional curvature such that the Gaussian curvature of $M$ does not change its sign. If there exists a parallel umbilical-free isoperimetric section on $M$, then $M$ is flat.

THEOREM 2. Let $M$ be a closed surface of a 4-dimensional Riemannian manifold $R^{4}$ of constant sectional curvature $c \leqq 0$ such that the Gaussian curvature of $M$ does not change its sign. If there exists a parallel nondegenerate minimal section on $M$, then the mean curvature vector of $M$ is parallel.

THEOREM 3. Let $M$ be a surface in an m-dimensional simply-connected complete Riemannian manifold $\boldsymbol{R}^{m}$ of constant sectional curvature $c$ such that the Gaussian curvature of $M$ is constant. If there exists a parallel isoperimetric section on $M$, then either $M$ is contained in a (small or great) hypersphere of $R^{m}$ or $M$ is flat.

THEOREM 4. Let $M$ be a surface in a 4-dimensional simply-connected complete Riemannian manifold $R^{4}$ of constant sectional curvature $c \leqq 0$ such that the Gaussian curvature of $M$ is constant. If there exists a parallel minimal section on $M$, then either $M$ is contained in a great hypersphere of $R^{4}$ or the mean curvature vector $H$ of $M$ is parallel and $M$ is flat.

AMS (MOS) subject classifications (1970). Primary 53A05, 53B25, 53C40.

Key words and phrases. Parallel, isoperimetric, minimal nondegenerate, geodesic, umbilical-free sections, mean curvature, product surface. 
The proofs of these four theorems use the theory of analytic functions.

From Theorems 2 and 4 we have immediately the following characterization theorems of standard flat tori in euclidean 4-space.

COROLlary 1. Let $M$ be a closed surface in a euclidean 4-space such that the Gaussian curvature of $M$ does not change its sign. Then $M$ is the product surface of two plane circles if and only if there exists a parallel nondegenerate minimal section on $M$ (Chen [2]).

COROllary 2. Let $M$ be a surface in a euclidean 4-space such that the Gaussian curvature of $M$ is constant. Then $M$ is an open piece of a product surface of two plane circles if and only if there exists a nongeodesic parallel minimal section on $M$.

The detailed proofs of these results will be published in the author's forthcoming book Geometry of submanifolds published by Marcel-Dekker Inc., New York.

REMARKS. 1. If $R^{m}$ is euclidean, Theorem 1 was also obtained by B. Wegner.

2. It was pointed out by H. B. Lawson that Corollary 2 of [1] should add the following assumption " $M$ is not minimal surface of any hypersphere of $E^{m}$ ". The author would like to express his thanks to Professor Lawson.

\section{REFERENCES}

1. B.-Y. Chen, Surfaces with parallel mean curvature vector, Bull. Amer. Math. Soc. 78 (1972), 709-710.

2. - A characterization of standard flat tori, Proc. Amer. Math. Soc. 37 (1973), $564-567$.

3. S.-T. Yau, Submanifolds with constant mean curvature (to appear).

Department of Mathematics, Michigan State University, East Lansing, Michigan 48823 